\title{
How to Keep Children Safe in Traffic: Find the Daredevils Early
}

\author{
Ulrich Hoffrage \\ Max Planck Institute for Human Development
}

Ralph Hertwig
University of Basel

\author{
Angelika Weber \\ University of Applied Sciences
}

Valerie M. Chase

Max Planck Institute for Human Development

\begin{abstract}
Crossing the street in front of oncoming vehicles poses serious danger to young children. But is each young pedestrian similarly at risk? The authors aimed to identify children who are particularly prone to making risky and potentially harmful crossing decisions. They used a simple game involving risk to classify 5- to 6-year-olds as risk takers or risk avoiders. Children classified as risk takers made more crossing decisions at a busy 1-way street than risk avoiders, tolerated shorter time intervals between initiation of the crossing decision and arrival of the next vehicle, and were more likely to cause a (hypothetical) accident. Finally, they made decisions more quickly than risk avoiders. The authors discuss the implication of these results for traffic safety programs.
\end{abstract}

One of the most common features of everyday life is also one of the most dangerous: traffic. In industrialized countries, traffic is one of the leading causes of death among children (Malek, Guyer, \& Lescohier, 1990; Pitt, Guyer, Hsieh, \& Malek, 1990). In 2002, almost one fourth of all children between the ages of 5 and 9 years who were killed in traffic accidents in the United States were pedestrians (National Center for Statistics and Analysis [NCSA], 2003). It is during this age range that children start making crossing decisions without adult supervision (e.g., while walking to school).

Although in many accidents children are innocent victims, there is also a considerable proportion in which the accident is due to children's behavior. The typical explanation for such accidents is that children have specific age-related limitations that make poor decisions as pedestrians more likely. Candidate limitations include generally lower cognitive ability (i.e., in the tradition of the Piagetian research on cognitive development; e.g., Sandels, 1975), lack of domain-specific knowledge (e.g., traffic-related knowledge; Bongard \& Winterfeld, 1977), perceptual disadvantages (e.g., limited peripheral vision; Sandels, 1975), immature visual

Ulrich Hoffrage and Valerie M. Chase, Center for Adaptive Behavior and Cognition, Max Planck Institute for Human Development, Berlin, Germany; Angelika Weber, Department of Social Work, University of Applied Sciences, Würzburg-Schweinfurt, Germany; Ralph Hertwig, Department of Psychology, University of Basel, Basel, Switzerland.

This research was supported by the German Science Foundation and was carried out at the Max Planck Institute for Psychological Research in Munich, Germany. We thank Nikolas Sanders, Rüdiger Trimpop, and Werner W. Wittmann for helpful comments and Christhard Gelau for the original program for the computer game. Special thanks go to Maria Zumbeel, Simone Hermes, Nicole Nonnenbroich, and Susanne Decker for running the study and Inga Galinowski and Arne Schwarz for technical support.

Correspondence concerning this article should be addressed to Ulrich Hoffrage, Max Planck Institute for Human Development, Lentzeallee 94, 14195 Berlin, Germany. E-mail: hoffrage@mpib-berlin.mpg.de search strategies (e.g., Whitebread \& Neilson, 2000), distractability (e.g., Dunbar, Hill, \& Lewis, 2001), and inferior physical and motor skills (e.g., controlling impulsive reactions; Briem \& Bengtsson, 2000; Pitcairn \& Edlmann, 2000).

To compensate for some of these limitations, most traffic training programs teach children about traffic environments in general and provide them with simple rules for making crossing decisions. We put forth the thesis that these training programs are doomed to be only moderately successful because they treat each child the same. Accident statistics, however, indicate that some children are more accident prone than others, that is, they are more frequently involved in traffic accidents than others (e.g., Christoffel et al., 1986). A plausible and yet rarely examined possibility is that a disposition to take or avoid risks contributes to a child's accident proneness. If so, this fact has an important implication: If there were tools for identifying children who are likely to take high risks leading to involvement in traffic accidents, then training programs could be specifically tailored for and allocated to those who are most in need of such training.

What do we know about the role of individual differences in children's risk proneness, and how have these differences been studied to date? Researchers have typically searched for predictors of risk taking by examining post hoc the personalities and behavior of children who have been repeatedly involved in accidents (in traffic accidents, e.g., Christoffel et al., 1986; in any kind of accident, e.g., Krall, 1953; Manheimer \& Mellinger, 1967; Viney, 1971). For instance, using interviews with parents, some researchers have tried to identify factors that distinguish such children from those who have never been involved in traffic or other types of accidents. With few exceptions (e.g., Pless \& Peckham, 1986), these studies have been based on retrospective data.

This line of research indicates that the typical accident-prone child tends to be hyperactive (e.g., Christoffel et al., 1986; Grützmacher, 2001; DiScala, Lescohier, Barthel, \& Li, 1998); impulsive, inattentive, and easily distractible (e.g., Manheimer \& Mellinger, 1967; Matheny, 1987); and has problems controlling his actions. In addition, he has an adventuresome spirit, that is, he 
tends to be extroverted, attention seeking, and aggressive (Krall, 1953; Manheimer \& Mellinger, 1967). In the context of the typical accident-prone child, we intentionally use gender-biased language because this child in fact tends to be male. Accident statistics in western countries, for example, demonstrate that boys are typically twice as likely to be involved in traffic accidents as girls (e.g., Assailly, 1997; Chapman, Wade, \& Foot, 1982). Indeed, concerning accident liability (in traffic and other situations), gender is often seen as a proxy for personality differences (Manheimer \& Mellinger, 1967) and with some reason: Boys score higher than girls on variables such as impulsiveness, disobedience, and (physical) aggressiveness (however, if the definition of aggression is expanded to include verbal aggression, this picture changes; Crick et al., 1998). Similarly, boys and men are generally more likely than girls and women to take risks in games and natural environments (Byrnes, Miller, \& Schafer, 1999; Chapman, Foot, \& Wade, 1980; Ginsburg \& Miller, 1982; Hudgens \& Fatkin, 1985; Slovic, 1966). To account for the gender differences in traffic accident rates, it has been suggested that boys are more exposed to traffic than girls (because of more outdoor activities; Hargreaves \& Davies, 1996; van der Molen, 1981). However, controlling carefully for traffic exposure time, Howarth, Routledge, and RepettoWright (1974) and Routledge, Repetto-Wright, and Howarth (1974) found no support for this explanation.

The gender differences suggest the existence of personality traits that are correlated with accident proneness. This, in turn, is potentially important because it would mean that we could identify risk takers prospectively rather than retrospectively (i.e., before rather than after an accident occurred). How can this be done? Perhaps we can exploit the fact that risky behavior in laboratory gambling tasks correlates with personality traits related to accident proneness (Miller \& Byrnes, 1997; Trimpop, 1994). For the present purpose, we assume that children have a disposition either to take or to avoid risks and that this disposition underlies behavior across different domains. Specifically, we suggest that a child's risk disposition, measured on the basis of behavior in simple laboratory tasks, is predictive of his or her behavior as a pedestrian (in a context involving risk). Many studies have explored children's risk-taking behavior in situations that approximate real traffic, for instance, by using videos (we will review this research in the Discussion). We explored children's behavior under even more realistic conditions: Children stood at the curb of a street and made online traffic decisions. If it turns out that risky behavior in the laboratory is predictive for risky behavior on the street, measures can be taken to reduce the chance of a risk-prone child being involved in a traffic accident.

Participants in our study were boys and girls aged 5 to 6 years. We focused on this age group because traffic safety is of particular concern when children first start crossing the street alone, typically at around age 6 (Bongard \& Winterfeld, 1977; Howarth et al., 1974).

\section{Predictions}

In what follows, we derive three predictions that concern the link between children's risky behavior in games and in traffic. We created a naturalistic traffic context, in which children stand at the curb of a busy street and are required to indicate when they consider crossing to be safe. If risky behavior in games translates into risky behavior in real-world traffic, then we can anticipate differences between risk takers and risk avoiders on three temporal measures. Figure 1 depicts the traffic situation and introduces the three time measures: (a) The gap size denotes the time interval between two vehicles A and B, that is, the difference between the time at which A's rear bumper passes the crossing point (where the child is standing) and the time at which B's front bumper reaches the crossing point; (b) the leeway time denotes the time interval between initiation of crossing (i.e., time at which the child first steps onto the response mat) and arrival of the next vehicle (i.e., time at which the next vehicle's front bumper reaches the crossing point); and (c) the decision time denotes the time interval between the beginning of a gap in traffic and initiation of crossing. Note that gap size equals the sum of decision time and leeway time (see Figure 1). We use the terms go decision and stay decision to refer to the decisions to cross or to remain at the curb during a given gap, respectively.

\section{Prediction 1: Risk Takers Will More Frequently Arrive at a Go Decision Than Risk Avoiders. This Difference Will Be Most Pronounced for Medium-Sized Gaps and Smaller for Short and Long Gaps}

Why do we anticipate the difference in crossing behavior to be most pronounced for medium-sized gaps? Simply, because individual differences tend to disappear in both very simple tasks (here, deciding whether to cross during long gaps) and very challenging tasks (here, deciding whether to cross during short gaps). To appreciate this argument, consider a group of people of varying degrees of fitness going on a hike. While walking around a lake, where the ground is flat and easily navigated, the group stays together. As soon as the tour heads for the mountains, the people spread out, with the best hikers in front and the less-skilled hikers falling behind. But when they reach a large boulder blocking the route, most will have to stop, and only a very few skilled climbers will be able to get past the obstacle. The hikers' differences in ability are most noticeable when the tour poses a medium amount

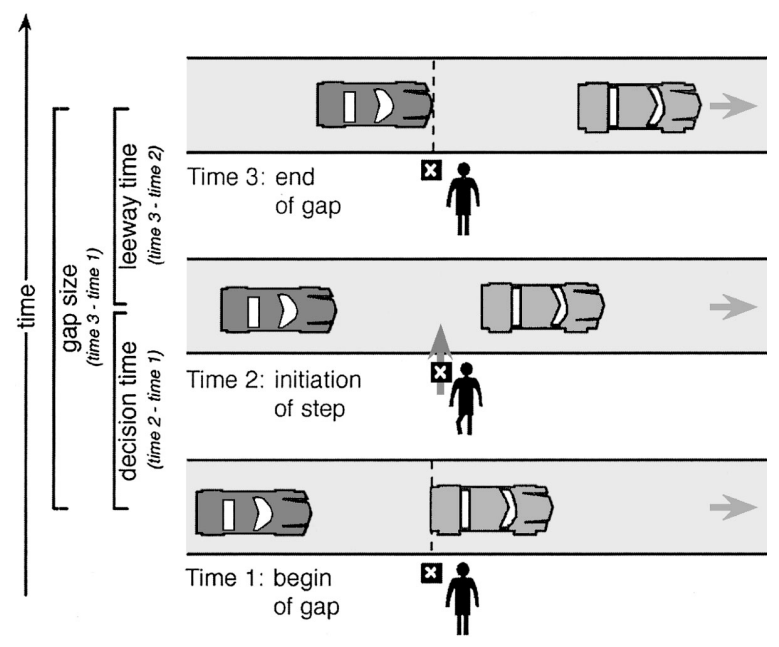

Figure 1. The real-traffic task and the three temporal measures on which the experimental predictions are based. Time passes from the bottom to the top, determining the direction in which this figure should be read. 
of difficulty, that is, on the mountain path, where the hikers fan out, but are hardly noticeable at the lake or the boulder. Using this analogy, we expect that children's difference in frequency of crossing decisions will be most pronounced when the gaps are of medium size (Similarly, Harrell, 1991, reported that differences in looking behavior of elderly pedestrians markedly increased for medium traffic volume.)

To determine whether a gap is long enough for a safe crossing, one estimates the time it takes to cross the street (crossing time) and the time until the approaching vehicle reaches the crossing point. Next, one determines whether the crossing time is shorter than the leeway time (i.e., the time interval between initiation of crossing and arrival of the next vehicle). For short gaps (and thus even shorter leeway times), crossing is extremely risky (the boulder, where everyone will stop), and for long gaps, crossing is hardly risky at all (the lake, where almost everyone can walk easily). In either of these cases, we do not expect the observable differences between risk takers and risk avoiders to be large. However, where uncertainty is greatest, that is, when gaps are of medium size, the two groups may detectably differ.

The hypothesized difference between risk takers and risk avoiders with respect to their go decisions may be due to several reasons. One reason directly refers to the possible mechanism underlying a go decision. At the core of this computational mechanism lies the comparison between the crossing time and the leeway time. To allow for a safe crossing, one should ensure that the crossing time be shorter than the leeway time, but just how much shorter? This question leads to the next prediction.

\section{Prediction 2: Risk Takers Tolerate Shorter Leeway Times Than Risk Avoiders}

We anticipate that a higher disposition for risk translates into a higher willingness to cross the street even if leeway time is short. As a consequence, the percentage of go decisions that leave a particular (or shorter) leeway time will be higher among risk takers than among risk avoiders. In fact, the distribution of leeway times a person is willing to accept when crossing the street may be used to indicate his or her risk disposition.

Leeway time is a particularly interesting notion because it also affords us the opportunity to calculate the (hypothetical) accident rate. Assuming that risk takers have a lower threshold for making a go decision, they will be more likely than risk avoiders to make a go decision even if a safe crossing is not possible. This leads to the following corollary of Prediction 2: Risk takers will have a higher hypothetical accidents rate than risk avoiders. Yet, this differences between risk takers and risk avoiders will be much smaller than the difference with respect to go decisions (during medium gaps; see Prediction 1). The reason is that for short leeway times, and thus for gaps in which a safe crossing is not possible, the uncertainty about whether one could cross is likely to be minimal.

\section{Prediction 3: Risk Takers Will Have Shorter Decision Times Than Risk Avoiders}

Although the hypothesis that risk takers tolerate shorter leeway times than risk avoiders would be sufficient to explain why they should also have a higher proportion of go decisions, still another factor may contribute to this difference hypothesized in Prediction 1. Risk takers might arrive more quickly at go decisions. Consider, for instance, a risk-taking and a risk-avoiding child, both of whom accept a leeway time of $6 \mathrm{~s}$. If their decision times are $1 \mathrm{~s}$ and $2 \mathrm{~s}$, respectively, then the risk taker could exploit a gap of $7.5 \mathrm{~s}$ to cross, whereas the (slower) risk avoider would probably reject it. Note that Prediction 3 is supported by the frequently reported observation that impulsiveness is closely related to risk-taking behavior (Miller \& Byrnes, 1997; Trimpop, 1994), where "impulsive" children are-by definition - those who respond more quickly and make more errors than "reflective" children (see Miller \& Byrnes, 1997).

\section{Method}

\section{Participants}

The participants in our study were 44 children from four Munich kindergartens. The children ( 22 boys, 22 girls) were randomly selected from a larger pool of children whose parents had given written permission for the children to take part in the experiment (originally we approached 55 parents of which 3 did not respond; of those who responded all gave us their permission). Children who participated ranged in age from 4 years 9 months to 6 years 5 months (mean age: 5 years 6 months).

\section{Materials}

The study required children to make crossing decisions in a real-traffic situation and to play two games. Each child completed the real-traffic task on one day (Day 1) and the two laboratory tasks, a gambling game and a computer game, on another day (Day 2).

Real-traffic task. The real-traffic task was similar to one of the tasks used by Demetre et al. (1992; the two-step task). Children stood at the curb of a one-way street in the center of Munich where there was no traffic light or crosswalk. (In 2002, 79\% of the young pedestrian fatalities in the United States occurred at nonintersection locations; NCSA, 2003.) They were instructed to monitor the traffic and indicate whenever they thought that a gap between two vehicles was wide enough to cross safely. To indicate a go decision, they had to step forward onto a mat that sent an electronic signal to a video camera on the other side of the street. The video camera, which was equipped with a counter that marked time in units of $40 \mathrm{~ms}$, was used to record the whole 8-min session for later analysis. Children were tested individually under the constant supervision of an experimenter. To ensure the children's safety, we required them to wear a harness with which the experimenter could hold them back if they tried to walk into the street. Prior to the test phase, children underwent a 2 -min practice phase. During the practice phase, all of them seemed to learn the task quickly and to grow accustomed to the harness (i.e., gave the impression that they had forgotten about it). At the end of the session on Day 1, they received a toy for their participation.

On Day 2 (usually within the same week), the same children were required to play two games either in the laboratory or at their kindergarten: a gambling game adapted from Slovic (1966) and a computer game simulating the real-traffic task adapted from Tränkle and Gelau (1992; for an earlier and similar program, see Sivak, Soler, \& Tränkle, 1989). Half of the children played the gambling game first and the other half played the computer game first.

Gambling game. Children were presented with an array of 10 small closed wooden boxes. They were told that nine contained stickers (which children in our sample prized highly) and that the 10th box had a "devil" in it. Children were told that they could open as many boxes as they wanted. If they came across a sticker, they could keep it. However, if they 
happened to select the devil box, they would lose all of the stickers they had won up to this point and the game was over.

Each child completed three trials. The devil was assigned to 1 of the 10 boxes randomly in each trial. It was pointed out to the children that they could increase the number of stickers won by opening more boxes, but that opening more boxes would also increase the chances of finding the devil and thus losing everything. After each box was chosen, they had to decide whether they wanted to continue the trial. All instructions were given in language appropriate to children of this age. To verify that children understood the rationale of the game, we asked them to review the procedure in their own words. Judging from their descriptions, no child misconstrued the game.

Computer game. With this task, we aimed to simulate the real-traffic scenario as closely as possible. Children were seated in front of a 17-in. computer monitor that depicted-from an aerial view - a stream of oncoming vehicles (cars, trucks, motorcycles) all driving at the same velocity along a one-way street. (The panels in Figure 1 correspond closely to what children saw.) The gaps between vehicles varied in size. With a key press, children could send a symbol representing a pedestrian across the street. As in the real-traffic task, children were instructed to have the pedestrian cross the street whenever they thought it was safe to do so. They were told that for every successful crossing they would receive a piece of candy, whereas for every accident they would lose three pieces. At the beginning of the game, children received an endowment of 30 candies. When a decision led to a collision, the display froze briefly and a beep was emitted. Afterward, the traffic continued to flow and children could resume playing. The task consisted of two 1-min practice trials, each followed by four test trials of 3 min each. The accident rate was defined as the percentage of all crossings (in the test trials) in which the child caused the pedestrian to collide with a vehicle.

\section{Results}

We begin with the classification of children as risk taking or risk avoiding according to their behavior in the game contexts. We then briefly report the overall results for the real-traffic task, and finally we turn to the specific tests of Predictions 1, 2, and 3.

\section{Classification of Risk Takers and Risk Avoiders}

We classified the children as either risk taking or risk avoiding on the basis of their behavior in (each of) the two games. In the gambling game, classification (henceforth, gambling classification) depended on when children chose to stop opening boxes. Opening five boxes is the optimal strategy, that is, the one with the highest expected outcome (see Slovic, 1966). If children opened more than five boxes in at least one of the three trials, then we classified them as risk takers. If they opened five or fewer than five boxes on all three trials and terminated the game voluntarily in at least one trial, they were classified as risk avoiders. By these criteria, 39 children could be unambiguously classified: 26 as risk takers and 13 as risk avoiders. Five children could not be classified because in all three trials they opened five or fewer than five boxes but unfortunately had to stop because they got the devil each time. We excluded the data of those children when comparing risk takers and risk avoiders as classified according to the gambling game. ${ }^{1}$

In the computer game, we were able to classify 38 children ( 2 children could not be tested with the computer game, 3 refused to play this game or refused to continue after some minutes, and a 6 th was not able to operate the key appropriately). We used two different classifications. One depended on the accident rate, that is, the percentage of decisions that caused the pedestrian to collide with a vehicle. The other depended on the number of candies the children received (or lost). The number of candies was highly negatively correlated (-.72) with the accident rate. For each measure, we split the group of children at the two medians and classified those with higher accident rates and fewer candies as risk takers and the remaining children as risk avoiders.

Interestingly, risk disposition in the gambling game was not correlated with risk disposition in the computer game. When the median split in the computer game was based on children's accident rate, the correlation was $-.04, \chi^{2}(1, N=33)=.06$, ns, and when it was based on the number of candies, we obtained a correlation of $.04, \chi^{2}(1, N=33)=.06, n s$. This surprisingly low correlation implied the possibility that only one of the two measures would prove to be a valid behavior in real-world traffic. Indeed, this turned out to be true. Only the gambling classification was a good predictor of children's behavior in the real-traffic situation. Therefore, we decided to focus on this classification when reporting the tests of our predictions.

The computer game's predictive failure was not caused by our classification method (i.e., we classified children as risk takers and risk avoiders on the basis of a median split of the continuous variables accident rate and number of candies). In fact, when we correlated both continuous variables with children's behavior in the traffic task, the resulting correlations were negligible. We explore possible reasons for why the computer game failed to predict real-world behavior in the Discussion. We now turn to the general results for the real-traffic task.

\section{Real-Traffic Task}

Traffic density. Children encountered a total of 2,876 traffic gaps; the average number of gaps encountered by each child during the 8-min test session was 65 ( $S D=13.5$, range: 39 to 95). The traffic on this street was dense: median gap size was 3.2 s. Forty-seven percent of gaps were shorter than $3 \mathrm{~s}, 74 \%$ were shorter than $7 \mathrm{~s}$, and $86 \%$ were shorter than $12 \mathrm{~s}$. Average vehicle velocity (approximately $40 \mathrm{~km} / \mathrm{h}$ or $25 \mathrm{mph}$ ) was fairly constant across children.

Crossing time. To define the minimum gap size that theoretically would allow for a safe crossing, we needed to estimate children's crossing time. To determine this empirically, we had a different group of children in the same age range cross the same street at a traffic light. On average, it took them $3 \mathrm{~s}$ to run and $7 \mathrm{~s}$ to walk across the street. For our real-traffic task, therefore, gaps of less than $3 \mathrm{~s}$ were assumed to prevent safe crossings, gaps of 3 to $7 \mathrm{~s}$ were assumed to allow somewhat safe crossings, and gaps of longer than $7 \mathrm{~s}$ were assumed to permit safe crossings.

Go decisions. Out of the total of 2,876 gaps, the children made 640 go decisions (22.3\%); during the remaining 2,236 gaps $(78.7 \%)$, they decided to stay. Thus, on average, each child indi-

\footnotetext{
${ }^{1}$ We also derived several measures that yielded more than two categories (risk takers vs. risk avoiders). Specifically, we used the number of boxes that children opened and the number of boxes that remained closed, either in all trials or in those in which children voluntarily stopped looking, and combined them by either taking the minimum, average, or maximum of opened (closed) boxes. Probably because of the relatively small number of trials, none of the multiple polychotomous measures led to effect sizes that were consistently and markedly larger than those observed on the basis of the dichotomous classification.
} 
cated about 12 go decisions per session ( $S D=7.2$, range: 5 to 36 ). The children in our study showed mostly conservative decisionmaking behavior: In just $3.2 \%$ of the gaps of less than $3 \mathrm{~s}$ did children make a go decision. For gaps from 3 to $7 \mathrm{~s}$, the percentage of go decisions was 14.8. Even for gaps of greater than $7 \mathrm{~s}$, children made a go decision during only $65.7 \%$ of the gaps, thereby missing $34.3 \%$ of the opportunities to cross when theoretically it would have been safe. Most of the go decisions (481 of $640 ; 75.2 \%$ ) were made during gaps of longer than $7 \mathrm{~s}$. This overall conservative decision-making behavior is in line with results of previous observational and experimental studies (e.g., Connelly, Isler, \& Parsonson, 1996; Demetre et al., 1992; Hoffmann, Payne, \& Prescott, 1980; Routledge, 1975). Children's conservative decision-making behavior suggests that we succeeded in creating a realistic crossing scenario.

\section{Prediction 1: Do Risk-Taking Children Make More Go Decisions, in Particular, During Medium Gaps?}

In a first step of the analysis, we examined the go decisions for risk-taking and risk-avoiding children (using the gambling classification) across the wide range of gaps. Owing to the relatively small number of gaps per unit (each of $40 \mathrm{~ms}$ ), we first categorized all gaps up to $10 \mathrm{~s}$ into 1-s intervals and gaps of more than $10 \mathrm{~s}$ into larger intervals, as depicted in Figure 2. As this figure shows, the gap sizes (the sum of go and stay decisions) seem to follow a Poisson distribution: Gaps shorter than $1 \mathrm{~s}$ are relatively rare, then there is a peak for gaps between 1 and $3 \mathrm{~s}$, and after this peak the sums of the curves almost monotonically decrease and eventually approximate the $x$-axes. The figure also shows that risk takers begin to make go decisions during gaps that are clearly rejected by risk avoiders. Moreover, the indifference point, that is, the point at which go and stay decisions are equally frequent, is much further to the left for risk takers than for risk avoiders.

In a second step of the analysis, we computed statistics for short, medium, and long gaps separately. How did we delineate medium from short and long gaps? Medium gap sizes are those where the uncertainty (of whether to cross) is maximal. In contrast, short and long gaps involve little uncertainty. At what gap size, then, is the degree of uncertainty maximal? We defined the point of maximum uncertainty as the gap size for which go decisions were as frequent as stay decisions, that is, gaps during which the percentage of go decisions is $50 \%$. If risk takers have a lower criterion than risk avoiders when judging whether a gap permits a safe crossing, then the gap size for which risk takers experience maximum uncertainty should be shorter than the corresponding gap size for risk avoiders. As expected, the gap size associated with maximal uncertainty was smaller for risk takers than for risk avoiders -7 and $12 \mathrm{~s}$, respectively. ${ }^{2}$ The region between these endpoints is analogous to the mountain path in the hiking example before the hikers encountered the boulder. It is here where it is easiest to distinguish the skilled from the unskilled hikers, and the risk avoiders from the risk takers. Thus, we took these endpoints to define the interval encompassing medium gap sizes, and we took intervals smaller than $7 \mathrm{~s}$ and longer than $12 \mathrm{~s}$ to be short and long gaps, respectively. It is interesting to note that a completely orthogonal approach to defining the gap size would have arrived at very similar time intervals. Had we taken children's time to cross into account, we would have defined short gaps as being narrower than the time
Risk Taker

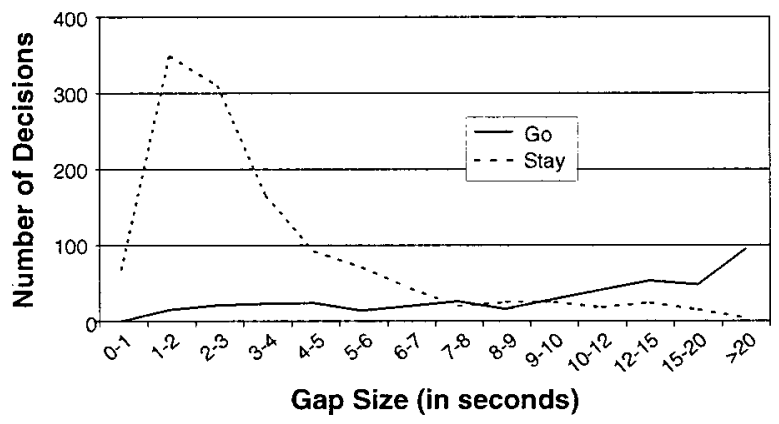

Risk Avoider

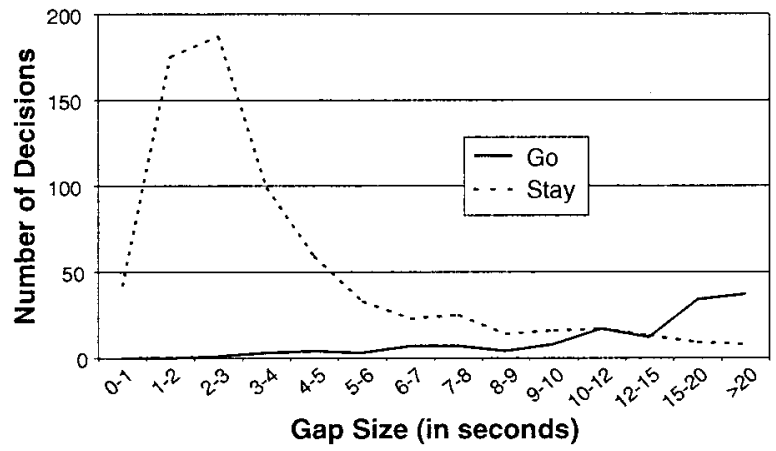

Figure 2. Number of go and stay decisions per gap-size interval, for risk takers and risk avoiders. Risk disposition has been determined by children's behavior in the gambling game. Within each panel, results are pooled across all children of this group. Because the 26 risk takers encountered more gaps than the 13 risk avoiders, the $y$-axes have been scaled such that the sum of go and stay decisions for both groups visually appears to be the same.

children needed to walk across the street (7 s) and long gaps as being $11 \mathrm{~s}$ or longer (i.e., $7 \mathrm{~s}$ to walk plus a safety interval of $50 \%$ - which is about the time children needed to run across the street).

Using these endpoints, 7 and $12 \mathrm{~s}$, we computed a child's percentage of go decisions for short, medium, and long gaps and then aggregated these numbers across all children (Table 1). The results confirm Prediction 1: For medium gaps, the difference between risk takers and risk avoiders in percentage of go decisions was almost twice as large as for short and long gaps. Note that according to Cohen (1988), effect sizes of $d=.2, .5$, and .8

\footnotetext{
${ }^{2}$ Specifically, we compared the proportion of go decisions in each pair of adjacent 1-s intervals to determine when this value surpassed $50 \%$ (i.e., the point of maximal uncertainty). Such comparisons have been performed for risk takers and for risk avoiders separately. For instance, risk takers (classified according to the gambling game) made go decisions in $32.8 \%$ of all gaps of a 6-s to 7-s duration and in $56.8 \%$ of all gaps of a 7-s to 8-s duration. For risk avoiders, the 1-s gap size intervals for which go and stay decisions were (about) equally frequent ranged from $10 \mathrm{~s}$ to $15 \mathrm{~s}$; we determined $12 \mathrm{~s}$ as the point of maximal uncertainty.
} 
Table 1

Percentage of Go Decisions During Short, Medium, and Long Gaps, Classified by the Gambling Game

\begin{tabular}{|c|c|c|c|c|c|c|c|c|c|}
\hline \multirow[b]{2}{*}{ Gap size } & \multicolumn{3}{|c|}{ Risk takers } & \multicolumn{3}{|c|}{ Risk avoiders } & \multicolumn{3}{|c|}{ Difference } \\
\hline & $\begin{array}{c}\text { Go decisions } \\
(\%)\end{array}$ & $S D$ & $S E$ & $\begin{array}{c}\text { Go decisions } \\
(\%)\end{array}$ & $S D$ & $S E$ & $\begin{array}{c}\text { Go decisions } \\
(\%)\end{array}$ & $t(d f)$ & $d$ \\
\hline Short $(x<7$ s $)$ & 12.5 & 21.4 & 4.2 & 2.8 & 3.3 & 0.9 & 9.7 & $2.25(27.31)^{\mathrm{a}, *}$ & .91 \\
\hline $\operatorname{Medium}(7 \mathrm{~s} \leq x<12 \mathrm{~s})$ & 58.4 & 28.3 & 5.6 & 40.3 & 33.8 & 9.4 & 18.1 & $1.76(37)^{*}$ & .61 \\
\hline Long $(12 \mathrm{~s} \leq x)$ & 81.5 & 22.5 & 4.4 & 75.1 & 16.2 & 16.2 & 6.4 & $0.91(37)$ & .32 \\
\hline
\end{tabular}

Note. Percentages have first been determined for each individual child and subsequently aggregated across all risk takers and risk avoiders.

a Whenever the degree of freedom is a fractional number, the null hypothesis "standard deviations are equal" had to be rejected and thus the degree of freedom has been adjusted accordingly.

$* p<.05$.

corresponds to small, medium and large effects, respectively. ${ }^{3}$ (When using the computer classification, we obtained no substantial or significant difference between risk takers and risk avoiders with respect to their go decisions.)

For the third step in our analysis of children's go decisions, we used a well-known statistical framework: signal detection theory (Green \& Swets, 1966). This framework can be applied to modeling any task in which an observer must detect signals (or items from a specific category) in the midst of noise (or items from another category). If one views crossing decisions as decisions regarding the presence of a signal, then the real-traffic task is just such a task: Children have to detect gaps that allow a safe crossing (go) and to reject gaps that cannot be safely used (stay). Crossing when it is safe can be considered a hit, and crossing when it is not safe a false alarm. In the framework of signal detection theory, a subjective decision criterion indicates how much risk a person is willing to accept to achieve a hit. If the cost of a false alarm is high compared with the cost of a miss (the miss rate is the complement of the hit rate), then this criterion should be conservative. The real-traffic task is such a case, because crossing when it is not safe (false alarm) may be fatal, whereas the cost of missing an opportunity to cross is typically minimal.

To determine the hit rate and the false alarm rate, one needs to identify what is a signal and what is noise in a specific environment. In our real-traffic task, this identification is not trivial because children (for good reasons) did not actually cross the street; thus we do not know for sure what would have been a safe gap (i.e., a signal). One might argue that $3 \mathrm{~s}$-the time children needed to run-may define what a signal is. However, we instructed children to indicate a go decision whenever they thought they could safely cross, thus leaving undefined for them what safely means. As it turned out, they considered most of the gaps of 3 or $4 \mathrm{~s}$, that is, gaps that left almost no room for error, as too short for safe crossings (see Figure 3). Nevertheless, to be able to identify signals, we assumed several temporal definitions of what counts as a safe gap. Specifically, we defined gaps longer than 3 (or $4,5, \ldots, 12$ ) $\mathrm{s}$ as safe. For each child and for each of those different sets of safe gaps, we computed the hit rate and the false alarm rate.

Table 2 displays the average hit and false alarm rates as a function of various definitions of what constitute safe gaps (i.e., signals). As expected, the specific values of both rates depend on the definition used. For instance, under the 3-s definition (mini- mum time it takes to run across the street), the 26 risk takers had an average hit rate of $45.4 \%$, whereas under the 12-s definition, their average hit rate was $81.5 \%$. For each of the definitions applied, risk takers had both a higher hit rate and a higher false alarm rate than risk avoiders. For instance, under the 7-s definition, risk avoiders had a hit rate of just $56.8 \%$, which means that they missed $43.2 \%$ of the gaps that were theoretically long enough for them to cross safely without having to run. Risk takers, in contrast, had a hit rate of $72 \%$, missing only $28 \%$ of the crossing opportunities under the 7-s definition. Table 2 also shows that-as expected-both groups' false alarm rates were low compared with the rate of these missed opportunities, which simply equals the complement of the hit rate. (Using the computer game classification, we discovered that the differences corresponding to those shown in Table 2 were negligible.)

To conclude, the results confirm Prediction 1: During gaps of all three sizes, and in particular during medium-sized gaps (between 7 and 12 s), risk takers arrived at a higher percentage of go decisions than risk avoiders (Table 1). Moreover, independent of how we defined a safe gap, risk takers also had both a higher hit rate (i.e., fewer missed opportunities) and a higher false alarm rate than risk avoiders (Table 2). As stated above, this observed dif-

\footnotetext{
${ }^{3}$ For none of the six entries in Table 1 ( 2 risk dispositions $\times 3$ gap-size intervals) did we find a significant difference between the first and the second half of gaps that the children encountered (the $t$ values ranged from 0.05 to 1.21 , and the effect sizes $d$ ranged from .02 to .50 ). A multivariate analysis of variance confirmed this result: Risk disposition as a between factor yielded the following: $F(1,38)=6.77, p<.05, \eta^{2}=.155$; gap-size interval (i.e., short, medium, and long) as a within factor yielded the following: $F(2,38)=96.14, p<.01, \eta^{2}=.722$; and first versus second half of gaps within the session as the second within factor yielded the following: $F(1,38)=2.7, n s, \eta^{2}=.068$ (the statistical power corresponding to this nonsignificant effect, however, was only .36). To get still another estimate of the effect sizes, we also applied Rosenthal, Rosnow, and Rubin's (2000) contrast and effect size approach. To test for the effect of gap size, we set the lambda weights for short, medium, and long gap size intervals to $-1,0$, and +1 , respectively, and obtained $r_{\text {contrast }}=0.91$ and $r_{\text {counternull }}=0.99$. As predicted, there was only a small effect of risk disposition (note that in this analysis the weight for medium gaps was 0 ): $r_{\text {contrast }}=0.06$ and $r_{\text {counternull }}=0.13$. When we used a quadratic function (with weights of $-1,2,-1$ ) to amplify the impact of gaps where the difference was predicted to be most pronounced, we obtained for the effect of risk disposition $r_{\text {contrast }}=0.18$ and $r_{\text {counternull }}=0.69$.
} 


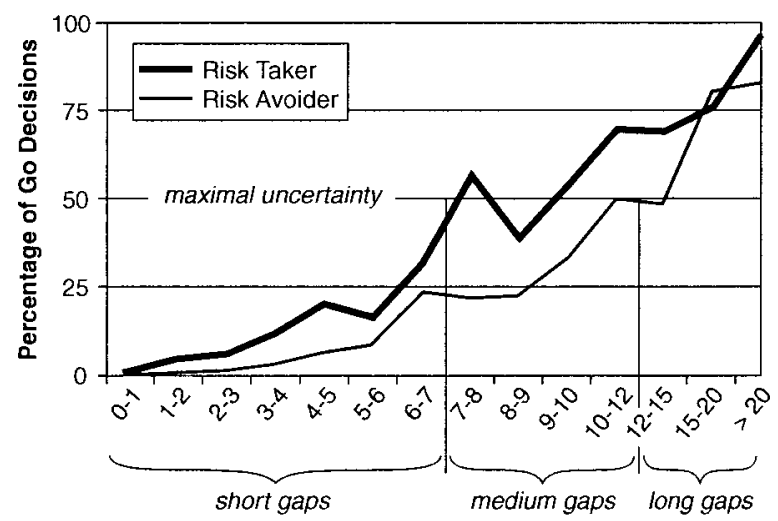

Gap Size (in seconds)

Figure 3. Percentage of go decisions per gap-size interval for children when classified according to behavior in the gambling game.

ference in crossing decisions between risk takers and risk avoiders can be attributed to risk takers tolerating shorter leeway times than risk avoiders (Prediction 2), to their deciding more quickly (Prediction 3 ), or to both.

\section{Prediction 2: Do Risk Takers Tolerate Shorter Leeway Times Than Risk Avoiders?}

To reiterate, leeway time is the time interval between initiation of crossing and the arrival of the next vehicle. Figure 4 shows the total number of go decisions that left a leeway time up to a particular duration divided by the total number of gaps encountered. Prediction 2 could be supported: Among children classified as risk takers, the percentage of go decisions leaving a leeway time of less than $1 \mathrm{~s}, 2 \mathrm{~s}, 3 \mathrm{~s}$, and so on up to $10 \mathrm{~s}$ was more than twice as high as for risk avoiders. For instance, children classified as risk takers encountered a total of 1,654 gaps and made a go decision

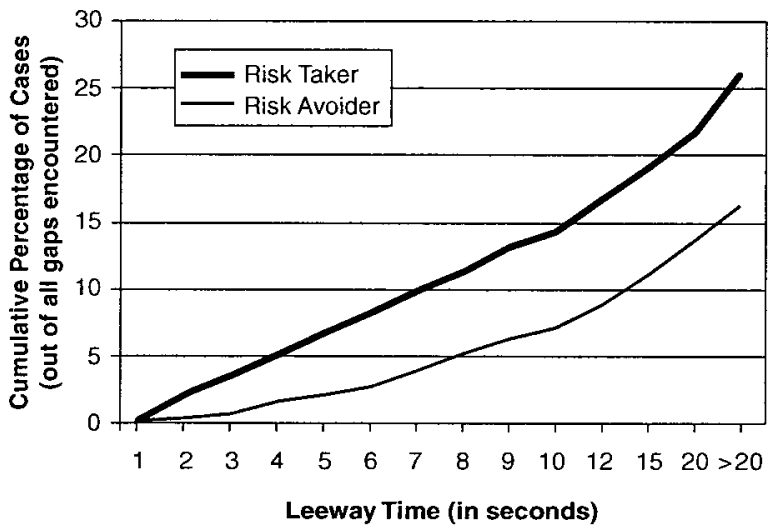

Figure 4. Cumulative percentage of cases leaving a particular (or shorter) leeway time. The figure shows the graphs for the gambling classifications.

that left a leeway time of less than $7 \mathrm{~s}$ in $162(9.8 \%)$ cases, whereas for risk avoiders, this value was only $3.9 \%(\Delta=5.9$ percentage points). (The differences between the two curves in Figure 4 cannot be explained by different distributions of gap sizes for the two groups - the distributions were almost identical.)

Although Figure 4 depicts the percentages based on all gaps the two groups encountered, the unit for the inferential statisticshere and for all other tests reported in this article-was a single child. The averages of all child-specific percentages of go decisions with a leeway time of less than $7 \mathrm{~s}$ were 11.6 and 4.0 for risk takers and risk avoiders, respectively ( $\Delta=7.5$ percentage points), $t(31.77)=2.3, p=<.05$ (one-sided),$d=.87$.

As a corollary of Prediction 2, we stated that risk takers will have a higher hypothetical accident rate than risk avoiders. For our real-traffic task, a leeway time of $3 \mathrm{~s}$ - the time children of this age needed to run across the street-was assumed to be the minimum time interval for a crossing (albeit a dangerous one). Thus, we

Table 2

Signal Detection Analysis of Go-Stay Decisions

\begin{tabular}{|c|c|c|c|c|c|c|c|c|c|c|c|c|c|c|}
\hline \multirow[b]{3}{*}{ Threshold $^{\mathrm{a}}$} & \multicolumn{7}{|c|}{ Hit rate $(\%)$} & \multicolumn{7}{|c|}{ False alarm rate $(\%)$} \\
\hline & \multicolumn{2}{|c|}{ Risk takers } & \multicolumn{2}{|c|}{ Risk avoiders } & \multirow[b]{2}{*}{ Difference } & \multirow[b]{2}{*}{$t^{\mathrm{b}}$} & \multirow[b]{2}{*}{$d$} & \multicolumn{2}{|c|}{ Risk takers } & \multicolumn{2}{|c|}{ Risk avoiders } & \multirow[b]{2}{*}{ Difference } & \multirow[b]{2}{*}{$t^{\mathrm{b}}$} & \multirow[b]{2}{*}{$d$} \\
\hline & $M$ & $S D$ & $M$ & $S D$ & & & & $M$ & $S D$ & $M$ & $S D$ & & & \\
\hline 3 & 45.4 & 20.1 & 31.0 & 13.8 & 14.4 & $2.32 *$ & .81 & 8.0 & 19.4 & 0.3 & 1.1 & 7.7 & $2.02 *$ & .85 \\
\hline 4 & 53.8 & 20.0 & 40.0 & 18.7 & 13.8 & $2.08 *$ & .73 & 9.6 & 20.9 & 1.0 & 1.5 & 8.6 & $2.10^{*}$ & .88 \\
\hline 5 & 59.9 & 20.3 & 47.3 & 20.5 & 12.6 & $1.83 *$ & .64 & 10.7 & 20.7 & 1.4 & 1.8 & 9.3 & $2.27^{*}$ & .95 \\
\hline 6 & 67.0 & 20.9 & 53.3 & 22.9 & 13.7 & $1.87 *$ & .65 & 11.4 & 21.3 & 1.8 & 2.3 & 9.7 & $2.28^{*}$ & .95 \\
\hline 7 & 72.0 & 20.8 & 56.8 & 22.2 & 15.2 & $2.11 *$ & .74 & 12.5 & 21.4 & 2.8 & 3.3 & 9.7 & $2.25^{*}$ & .91 \\
\hline 8 & 74.0 & 20.4 & 62.0 & 21.1 & 11.9 & $1.70 *$ & .59 & 14.2 & 21.9 & 3.9 & 4.6 & 10.3 & $2.31^{*}$ & .91 \\
\hline 9 & 77.4 & 20.2 & 65.3 & 19.7 & 12.2 & $1.79 *$ & .62 & 15.1 & 21.5 & 4.4 & 5.2 & 10.7 & $2.40^{*}$ & .93 \\
\hline 10 & 79.8 & 19.2 & 69.2 & 16.4 & 10.6 & $1.70 *$ & .59 & 16.6 & 21.6 & 5.3 & 5.0 & 11.2 & $2.52 * *$ & .98 \\
\hline 11 & 80.5 & 22.1 & 72.1 & 15.3 & 8.4 & 1.22 & .43 & 17.5 & 21.4 & 6.5 & 5.6 & 11.1 & $2.47 * *$ & .94 \\
\hline 12 & 81.5 & 22.5 & 75.1 & 16.2 & 6.4 & 0.91 & .32 & 18.6 & 21.2 & 7.3 & 5.9 & 11.3 & $2.52 * *$ & .95 \\
\hline
\end{tabular}

Note. Averaged child-specific hit rate and false alarm rates. The threshold, that is, the minimum gap size considered as long enough for a safe crossing, defines what counts as a signal. The hit rate is defined as the percentage of go decisions during gaps exceeding the threshold, and the false alarm rate is defined as the percentage of go decisions during gaps shorter than the threshold.

${ }^{\mathrm{a}}$ In seconds. ${ }^{\mathrm{b}}$ All $t \mathrm{~s}$ for the comparison of hit rates had $d f=37$; all $t$ s for the comparison of false alarm rates had $d f \mathrm{~s}$ between 25.33 and 31.81 .

$* p<.05$. ** $p<.01$. 
defined the hypothetical accident rate as the percentage of all gaps during which a go decision that left a leeway time of less than $3 \mathrm{~s}$ was made. In our study, the overall hypothetical accident rate was $2.7 \%$ (78 of 2,876 ). We cannot ascertain whether a go decision with a leeway time of less than $3 \mathrm{~s}$ would truly have resulted in an accident: Although the child indicated a go decision, he or she might have stepped back at the last moment and, likewise, the driver might have slowed down to avoid collision. Moreover, some go decisions left such a short leeway time (e.g., $40 \mathrm{~ms}$ ) that one may assume the child was aiming for the gap after the car in front of him. Thus, the real accident rate would probably be lower than the hypothetical accident rate (we certainly hope so). But the hypothetical accident rate still provides us with useful information in that it highlights the differences between risk takers and risk avoiders.

Risk takers indeed had a significantly higher hypothetical accident rate $(3.7 \%$; 61 of 1,654$)$ than risk avoiders $(0.6 \% ; 5$ of 857$)$. This difference appears to be small. However, when viewed in terms of Rosenthal and Rubin's (1982) binomial effect-size display, it amounts to a difference of 9 percentage points between the two groups. This difference is substantial, in particular when the dependent variable implies vital consequences (for a discussion of when and why small correlations imply important effects, see Rosenthal \& Rosnow, 1991). The averages of children's individual hypothetical accident rates were 4.56 and 0.68 for risk takers and risk avoiders, respectively ( $\Delta=3.88$ percentage points), $t(28.81)=2.34, p<.05$ (one-sided),$d=.92$. One may argue that an accident can only occur once a go decision is made. Thus, as an alternative measure, one can relate the number of crossings that left a particular leeway time to the total number of go decisions (rather than to the total number of all gaps). In general, this measure yields higher percentages and higher absolute differences. For instance, the hypothetical accident rates amount to $14.4 \%$ (61 of 425 ) versus $3.6 \%$ ( 5 of 137), which corresponds to a difference of 14 percentage points in the binomial effect-size display. (The corresponding curves for Figure 4 based on the computer classification were almost identical for risk takers and risk avoiders.)

To summarize, consistent with Prediction 2, risk takers (using the gambling classification) tolerated shorter leeway times than risk avoiders. Risk takers also had a higher hypothetical accident rate, that is, a higher percentage of go decisions (out of all gaps) that left a leeway time of less than $3 \mathrm{~s}$-the time children needed to run across the street.

\section{Prediction 3: Do Risk-Taking Children Reach Crossing Decisions More Quickly?}

The average decision time between the beginning of a gap and the child's initiation of crossing (i.e., a step forward onto the signal mat) was $2.32 \mathrm{~s}$. However, the distribution of decision times was quite skewed (in $70.1 \%$ of cases, the decision time was below this average) and strongly affected by outliers (of up to $23 \mathrm{~s}$ ). Because such outliers seem less likely to stem from risk disposition than from fluctuations in children's attention, we used median values to test Prediction 3. After computing the median decision time for each child individually, we compared the means of these medians for risk takers and risk avoiders. As predicted, the average median was lower for risk-taking than for risk-avoiding children (1.6 s and $2.6 \mathrm{~s}$, respectively), $t(15.66)=2.34, p<.05$ (one-sided), $d=$
1.25. Note that the decision time was a function of the gap size. Specifically, the average decision times (and their standard deviations) for short, medium, and long gaps were $0.90(0.75) \mathrm{s}, 1.83$ (1.55) s, and $3.3(3.60) \mathrm{s}$, respectively. That is, the longer the gap, the more time children took to decide $(r=.32, p<.01$; the corresponding correlations within the group of short, medium, and long gaps were $.15, .12$, and .09 , respectively; $p<.05$, $n s$, and $p<$ .05 , respectively). Within each single gap-size interval (short, medium, and long), however, risk takers required less time than risk avoiders to make their decisions. (The computer classification yielded a small and not significant difference in the direction opposite to the prediction.)

\section{Discussion}

We found evidence that children's risk-taking behavior is correlated across different domains. Specifically, we observed children's behavior in two games involving risk and uncertainty and in an everyday-traffic situation. Risk taking in one of the games, the gambling game, proved to be predictive of risk taking in the everyday-traffic context.

The difference between risk takers and risk avoiders in the real-traffic task was largest during medium-sized gaps (7 to $12 \mathrm{~s}$ ), when uncertainty about the possibility of a safe crossing was highest. During these gaps, risk takers (as classified in the gambling game) made a higher proportion of go decisions than risk avoiders. Two factors contribute to this finding: Risk takers are willing to tolerate shorter leeway times than risk avoiders (Prediction 2), and they reached their crossing decisions more quickly (Prediction 3).

The most pronounced difference between risk takers and avoiders was found in a range of leeway times in which there was little danger of an accident (over $5 \mathrm{~s}$ ). With respect to gaps that left a leeway time so short that it was very dangerous to cross (less than $3 \mathrm{~s}$ ), the difference between groups shrank but did not disappear. We suggest that even such a small difference may be consequential in light of the numerous crossing decisions children need to make everyday. We now discuss how our findings relate to previous research and how they can be instrumental in improving traffic safety programs.

\section{Previous Research}

Although its assumptions have often been criticized (e.g., Baillargeon, 1993), Piaget's theory of cognitive development is still the major source of explanations of age-related limitations in cognitive abilities, some of which appear to be critical in traffic (e.g., Coppens, 1986; Demetre, 1997; Hargreaves \& Davies, 1996; Sandels, 1975). Take, for instance, young children's (relative) lack of ability to integrate two or more variables (e.g., object distance and speed) into a single judgment (e.g., time to arrive). Piaget and others showed that preoperational children (under age 7) have difficulties performing such integration of variables (Cross, 1988; Piaget, 1970; Siegler \& Richards, 1979).

In a review of approaches to traffic education for young children, Vinjé (1981) systematically linked age-related cognitive abilities (or lack thereof) to the specific abilities necessary to cross a street safely. She concluded that compared with adults, children exhibit deficits in perception (e.g., because of their shorter stature 
and lower visual acuity), attention (e.g., higher distractability), and motor and cognitive abilities (e.g., lower memory capacity) that might put them at risk in traffic. However, Vinjé (1981) was skeptical about the extent to which such age-related limitations in cognitive abilities can explain young children's accident proneness in traffic, pointing out that "the type of tasks concerned is often far removed from the reality of traffic participation" (p. 236).

To redress this situation, Vinjé $(1982 a, 1982 b)$ and other researchers studied children's performance on laboratory tasks that approximated real-world traffic (Bongard \& Winterfeld, 1977; Briem \& Bengtsson, 2000; Cambon de Lavalette \& Laya, 1988; Günther \& Limbourg, 1976; Hoffmann et al., 1980; Pitcairn \& Edlmann, 2000; Whitebread \& Neilson, 2000). For instance, in some studies, children were presented with slides or film sequences of real-world traffic situations and asked to predict the arrival time of a vehicle or judge the possibility for a safe crossing (Cambon de Lavalette \& Laya, 1988; Hoffmann et al., 1980; Pitcairn \& Edlmann, 2000; Vinjé, 1982a, 1982b; Whitebread \& Neilson, 2000). Across these studies, children made more errors than adults. For example, Vinjé (1982a, 1982b) conducted one study in which children and adults were shown a film featuring a pedestrian in a real-world traffic situation. Whereas $88 \%$ of 7 -yearolds misjudged whether the pedestrian could safely cross the street, only $21 \%$ of adults gave incorrect judgments.

Although such simulations capture some aspects of real-world traffic, their external validity is still limited. For instance, if on the basis of Vinjé's (1982a, 1982b) study one predicted that more than 7 out of 8 children's crossing decisions are wrong, one would grossly overestimate the actual traffic accident rate for children in corresponding real-traffic situations. Similarly, one would overestimate the accident rate for adults on the basis of the finding that one fifth of them incorrectly judged the possibility of safely crossing. These very high error rates may mean that even traffic simulations do not afford all of the relevant information and cues available in the real world, or that participants do not expend much effort in such situations because they do not believe that their decisions matter (for a direct comparison between laboratory and field analyses of decisions involving risk, see Ebbesen, Parker, \& Konecni, 1977).

Some researchers have tried to ensure external validity by observing children's behavior in real-world traffic environments (e.g., Ampofo-Boateng et al., 1993; Briem \& Bengtsson, 2000; Zeedyk, Wallace, \& Spry, 2002; for a review, see van der Molen, 1981). Here the focus is on finding out how children actually behave in traffic and to what extent they obey traffic safety rules (e.g., stop at the curb and look both ways before crossing). The assumption underlying most training programs is that children have insufficient knowledge of or experience with traffic safety rules. Contrary to this assumption, the surprising finding of these observational studies is that younger children (5-6 years) seem to know basic traffic rules quite well (e.g., Bongard \& Winterfeld, 1977) and sometimes follow the rules even more conscientiously than do older children and adults. Moreover, they appear to be more conservative in their crossing decisions (i.e., to reject gaps that older pedestrians would accept; Connelly et al., 1996; Hoffmann et al., 1980; Thomson, 1991; van der Molen, 1981).

Taken together, the results obtained from laboratory studies exploring limitations in cognitive abilities and from observations of children's behavior in real-world traffic situations do not pro- vide a completely satisfying explanation of why children are more at risk than adults when crossing the street. Adopting a different approach, we did not deal with general developmental constraints (or lack of skills). We turned to an important practical question, namely, how to identify children who are at higher risk of being involved in traffic accidents? We answered this question by implementing a real-world traffic setting, using the two-step task whose validity Demetre et al. (1992) have demonstrated quite carefully. This naturalistic approach required considerable expense and equipment, which may explain why it is rarely used. We believe that the investment has paid off: Our findings suggest that it is possible to identify the daredevils - children willing to take more risks showed this behavior not only in a gambling game but also in real-world traffic. It remains to be seen how robust our findings are. One may, for instance, wonder to what extent they generalize to other traffic situations and locations. Previous research suggests that some traffic-related behavior, for instance, looking behavior of adults, changes across locations (Job, Haynes, Prabhakar, Lee, \& Quach, 1998; Wilson \& Grayson, 1980). There is, however, reason to believe that risk-taking behavior is more robust across situations and locations than head movements, which are highly contingent on the visual scenario. Before we discuss the implications of our results for safety training programs, we first demonstrate that the daredevils' risk disposition cannot simply be explained by their gender, and we discuss how they can be identified.

\section{Who Are the Daredevils?}

They are the boys, right? In fact, we observed several behavioral differences between boys and girls in the real-traffic task. First, boys made more go decisions than girls: $23.9 \%$ (316 of 1,320) versus $20.8 \%$ (324 of 1,556). Second, they had a higher percentage of go decisions with a leeway time of less than $7 \mathrm{~s}: 9.0 \%$ (118 of $1,320)$ versus $7.0 \%(109$ of 1,556$)$. Third, the hypothetical accident rate was higher: $3.3 \%$ (43 of 1,320 ) versus $2.0 \%$ (31 of 1,556). These results are in line with traffic accident statistics that consistently report a higher accident rate for boys than for girls (e.g., NCSA, 2003). Still, the gender differences we observed are dwarfed by the observed differences between risk takers and risk avoiders, suggesting that the latter cannot simply be reduced to gender. Moreover, boys were not overrepresented among risk takers (using the gambling classification): 7 of 13 risk avoiders and 13 of the 26 risk takers were boys $(r=.036, n s)$.

Are daredevils those children who lack traffic-related knowledge? To examine for this possibility, we interviewed children in our sample about some basic traffic rules (e.g., how to behave at traffic lights or pedestrian crosswalks). Somewhat to our surprise, we found no relation between their amount of knowledge and their behavior in the real-traffic task. Of course, this finding does not rule out that children's domain-specific knowledge determines other behaviors such as choosing a good location to cross. A recent study by Zeedyk, Wallace, Carcary, Jones, and Larter (2001), however, puts even this link in doubt. In their study, 5-year-old primary school children participated in a program that taught which locations are safe (vs. dangerous) when crossing a street. Although the training succeeded in increasing their knowledge, children who received such training performed no better in a real-traffic environment than children in a control group. 


\section{How Do We Identify the Daredevils?}

Given the level of present knowledge and the fact that ours is one of the first attempts to measure children's risk disposition and examine whether it accounts for their risk-taking and risk-avoiding behavior as pedestrians, we intentionally selected two very different decision tasks (involving risk) to measure risk disposition. In other words, in the selection of "risk meters," we took a leap of faith. We now know that only one of the two risk meters proved to be an excellent predictor of real-world traffic behavior. With the benefit of hindsight, we also can suggest an explanation for why behavior in the gamble but not in the computer game is correlated with real-world behavior. In the computer game, successful performance (i.e., maximum number of successful crossings) involves a trade-off between hits and false alarms. Although false alarms (resulting in accidents) result in loss of points, these losses can be compensated for by more hits (i.e., successful crossings). In the gambling game, in contrast, all rewards a person acquires in the game will be lost by one wrong move (i.e., hitting the box with the devil). Thus, the computer game and the gambling game's payoff structures were completely different, and the gambling game's payoff structure is in fact a scaled-down version of the traffic task's payoff structure. As in the gambling game, risky decisions in the traffic task entail noncompensatory and large losses. This large disutility of accidents is, for instance, reflected in the high miss rates and low false alarm rate in the real-traffic task (see Table 2).

This discussion suggests that the computer game would be more predictive if it were implemented differently. We propose that in future investigations the payoff structure ought to better mimic the much higher costs of wrong decisions in traffic. In addition, children should receive feedback in a more transparent way. In our program, the scores for safe crossings and accidents, and the corresponding candies, were hidden from the children so as not to distract them and were only given to them afterward. This procedure, however, may have led some of them to forget about the consequences of risky behavior: Sixty-three percent (median = $66 \%$, range $16 \%-93 \%$ ) of their crossing decisions resulted in an accident. Providing them with feedback online may lower their accident rates and lead to more careful behavior. Another reason why the computer game should not be written off prematurely is that it allows researchers to manipulate the difficulty of the task systematically (i.e., by using different gap sizes and by changing the traffic flow). Changing difficulty systematically may prove to be helpful in distinguishing risk takers from risk avoiders. Finally, the computer game renders possible a signal detection analysis, and this, in turn, allows one to distinguish between skill and risk disposition.

Unlike the computer game, the gambling game turned out to be a predictive risk meter. However, this is not to say that our implementation could not be optimized. Clearly, it can be. For instance, in the gambling task, we used only three trials. Moreover, we deliberately choose not to deceive children, that is, the devil was randomly placed under the boxes, and thus some children were doomed to find the devil before they reached the optimal number of boxes. Those trials were uninformative because they did not distinguish between risk takers and risk avoiders. As a consequence, we based the risk classification on a relatively small number of trials, namely, a total of 81 trials or an average of 1.8 trials per child. Clearly, using more trials in future studies is likely to increase the reliability of the risk-disposition classification. It would also allow researchers to operationalize risk disposition as a continuous (Lejuez et al., 2002) rather than a dichotomous variable, thereby probably increasing the effect sizes obtained (MacCallum, Zhang, Preacher, \& Rucker, 2002).

We only selected two risk meters - there are others. In their balloon analogue risk task, Lejuez et al. (2002), for instance, used a game that is similar to our gambling task. Adult participants inflated a ball by pumping air into it (simulated on a computer screen). With every pump, they gained money. However, with each pump participants took the risk of causing the ball to explode, and because each pump had the same prior probability of causing an explosion, the likelihood of explosion increased with the number of pumps. If it happened, all of the money accumulated was lost (akin to our gambling task). Lejuez et al. observed that the number of pumps, that is, the amount of risk taken, significantly correlated with various self-reported risky behaviors. We suspect that this game, as did our gambling game, would also engage young children.

To conclude, we believe that researchers are only at the beginning of designing reliable and valid risk meters. Such meters should require a minimum of equipment, be easy to administer, and engage young children. The gambling task is a promising starting point that has the potential to be integrated into training programs. In addition to risk disposition, such training programs may also monitor other variables, such as general intelligence, other personality dispositions (e.g., Lejuez et al., 2002), and problem behavior (West, Train, Junger, West, \& Pickering, 1999).

\section{Implications for Traffic Safety Programs}

Traditionally, training programs have taught children to follow general safety rules (e.g., to cross only when no vehicle is in sight). In the context of simulated traffic settings (e.g., in the classroom, in films, or in books), these rules are typically verbally communicated. Unfortunately, the efficacy of such road safety programs is poor (e.g., Ampofo-Boateng \& Thomson, 1989; Luria, Smith, \& Chapman, 2000; Malek et al., 1990; Thomson, 1991; van Schagen \& Rothengatter, 1997). One suggested remedy is to teach rules of thumb, such as only cross when no vehicle is in sight in real-life settings. In fact, a study by van Schagen (1988) suggested that 7-year-old children benefit from systematic training on crossing decisions in real-traffic situations. In a recent review of 15 randomized control trials on safety education of pedestrians (14 trials targeted children), Duperrex, Bunn, and Roberts (2002) concluded that such programs have the potential to change children's knowledge and observed behavior (though, as they also point out, their effects on injury rates are unknown). Moreover, Thomson (1991) argued that providing online feedback is by far the most promising approach to road safety training (see also Ampofo-Boateng et al., 1993; Connelly et al., 1996).

The results of our study suggest another important determinant of an effective training program. We believe that training is more effective the more it succeeds in identifying those who are prone to risky behavior. In addition, if we succeed in identifying the mechanisms that makes risk takers accept higher levels of riskfor instance, by accepting shorter leeway times (see Prediction 2) - we can design and implement training programs tailored to 
those mechanisms. For instance, risk takers may profit from training in visual timing skills (e.g., in the "pretend road" task, Young \& Lee, 1987; in the two-step task, Demetre et al., 1992; or by the method introduced by van Schagen, 1988). On the basis of behavioral feedback, children's timing still could be (re)calibrated, thus resulting in more conservative crossing decisions. In this sense, the results obtained in our study warrant optimism that educators - be they parents, teachers, or psychologists - will have some chance to find and teach the daredevils.

\section{References}

Ampofo-Boateng, K., \& Thomson, J. A. (1989). Child pedestrian accidents: A case for preventive medicine. Health Education Research, 5, 265-274.

Ampofo-Boateng, K., Thomson, J. A., Grieve, R., Pitcairn, T., Lee, D. N., \& Demetre, J. D. (1993). A developmental and training study of children's ability to find safe routes to cross the road. British Journal of Developmental Psychology, 11, 31-45.

Assailly, J. P. (1997). Characterization and prevention of child pedestrian accidents: An overview. Journal of Applied Developmental Psychology, 18, 257-262.

Baillargeon, R. (1993). The object concept revisited: New direction in the investigation of infants' physical knowledge. In C. Granrud (Ed.), Visual perception and cognition in infancy. Carnegie Mellon Symposia on Cognition (pp. 265-315). Hillsdale, NJ: Erlbaum.

Bongard, A. E., \& Winterfeld, U. (1977). Verkehrswissen und Verkehrsverständnis bei fünf- bis sechsjährigen Kindern [5-6-year-old children's knowledge and understanding of traffic]. In Bundesanstalt für Straßenwesen (Hrsg.), Unfall- und Sicherheitsforschung Straßenverkehr (Bd. 13). Cologne, Germany: Bundesanstalt für Straßenwesen.

Briem, V., \& Bengtsson, H. (2000). Cognition and character traits as determinants of young children's behaviour in traffic situations. International Journal of Behavioral Development, 24, 492-505.

Byrnes, J. P., Miller, D. C., \& Schafer, W. D. (1999). Gender differences in risk taking: A meta-analysis. Psychological Bulletin, 125, 367-383.

Cambon de Lavalette, B., \& Laya, O. (1988). The role of education in the prevention of pedestrian accidents among children. In T. Rothengatter \& R. de Bruin (Eds.), Road user behaviour: Theory and research (pp. 466-472). Assen/Maastricht, the Netherlands: Van Gorcum.

Chapman, A. J., Foot, H. C., \& Wade, F. M. (1980). Children at play. In D. J. Oborne \& J. A. Levis (Eds.), Human factors in transport research: Vol. 2. User factors: Comfort, the environment and behaviour (pp. 380-388). London: Academic Press.

Chapman, A. J., Wade, F. M., \& Foot, H. C. (Eds.). (1982). Pedestrian accidents. Chichester, England: Wiley.

Christoffel, K. K., Schofer, J. L., Jovanis, P. P., Brandt, B., White, B., \& Tanz, R. (1986). Childhood pedestrian injury: A pilot study concerning etiology. Accident Analysis \& Prevention, 18, 25-35.

Cohen, J. (1988). Statistical power analysis for the behavioral sciences. Hillsdale, NJ: Erlbaum.

Connelly, M. L., Isler, R., \& Parsonson, B. S. (1996). Child pedestrian's judgments of safe crossing gaps at three different vehicle approach speeds: A preliminary study. Education and Treatment of Children, 19, $19-29$.

Coppens, N. M. (1986). Cognitive characteristics as predictors of children's understanding of safety and prevention. Journal of Pediatric Psychology, 11, 189-202.

Crick, N. R., Werner, N. E., Casas, J. F., O’Brien, K. M., Nelson, D. A., Grotpeter, J. K., \& Markon, K. (1998). Childhood aggression and gender: A new look at an old problem. In D. Bernstein (Ed.), Nebraska Symposium on Motivation (Vol. 45, pp. 75-141). Lincoln: University of Nebraska Press.

Cross, R. (1988). Application of children's concepts of speed at the kerbside: Accident vulnerability and implications for the teaching of science to young children. In T. Rothengatter \& R. de Bruin (Eds.), Road user behaviour: Theory and research (pp. 473-481). Assen/Maastricht, the Netherlands: Van Gorcum.

Demetre, J. D. (1997). Applying developmental psychology to children's road safety: Problems and prospects. Journal of Applied Developmental Psychology, 18, 263-270.

Demetre, J. D., Lee, D. N., Pitcairn, T. K., Grieve, R., Thomson, J. A., \& Ampofo-Boateng, K. (1992). Errors in young children's decisions about traffic gaps: Experiments with roadside simulations. British Journal of Psychology, 83, 189-202.

DiScala, C., Lescohier, I., Barthel, M., \& Li, G. (1998). Injuries to children with attention deficit hyperactivity disorder. Pediatrics, 102, 14151420.

Dunbar, G., Hill, R., \& Lewis, V. (2001). Children's attentional skills and road behavior. Journal of Experimental Psychology: Applied, 7, $227-$ 234.

Duperrex, O., Bunn, F., \& Roberts, I. (2002). Safety education of pedestrians for injury prevention: A systematic review of randomised controlled trials. British Medical Journal, 324, 1129-1130.

Ebbesen, E. B., Parker, S., \& Konecni, V. J. (1977). Laboratory and field analyses of decisions involving risk. Journal of Experimental Psychology: Human Perception and Performance, 3, 576-589.

Ginsburg, H. J., \& Miller, S. M. (1982). Sex differences in children's risk-taking behavior. Child Development, 53, 426-428

Green, D. M., \& Swets, J. A. (1966). Signal detection theory and psychophysics. New York: Wiley.

Grützmacher, H. (2001). Unfallgefährdung bei Aufmerksamkeits- und Hyperaktivitätsstörung [Risk of accidents in children with attentiondeficit hyperactivity disorder]. Deutsches Ärzteblatt, 34/35, 524-526.

Günther, R., \& Limbourg, M. (1976). Erlebnis- und Verhaltensformen von Kindern im Straßenverkehr. Teil A: Dimensionen der Verkehrswelt von Kindern [Types of children's experience and behavior in traffic. Part A: Dimensions of traffic from children's perspective]. In Unfall und Sicherheitsforschung Straßenverkehr (Bd. 4, pp. 13-80). Cologne, Germany: Bundesanstalt für Straßenwesen.

Hargreaves, D. J., \& Davies, G. M. (1996). The development of risk-taking in children. Current Psychology, 15, 14-29.

Harrell, W. A. (1991). Precautionary street crossing by elderly pedestrians International Journal of Aging and Human Development, 32, 65-80.

Hoffmann, E. R., Payne, A., \& Prescott, S. (1980). Children's estimates of vehicle approach times. Human Factors, 22, 235-240.

Howarth, C. I., Routledge, D. A., \& Repetto-Wright, R. (1974). An analysis of road accidents involving child pedestrians. Ergonomics, 17, 319-330.

Hudgens, G. A., \& Fatkin, L. T. (1985). Sex differences in risk taking: Repeated sessions on a computer-simulated task. The Journal of Psychology, 119, 197-206.

Job, R. F. S., Haynes, J., Prabhakar, T., Lee, S. H. V., \& Quach, J. (1998). Pedestrians at traffic light controlled intersections: Crossing behaviour in the elderly and non-elderly. In K. Smith, B. G. Aitken, \& R. H. Grzebieta (Eds.), Proceedings of the Conference on Pedestrian Safety (pp. 3-11). Canberra, Australia: Australian College of Safety and Federal Office of Road Safety.

Krall, V. (1953). Personality characteristics of accident repeating children. Journal of Abnormal and Social Psychology, 48, 99-107.

Lejuez, C. W., Read, J. P., Kahler, C. W., Richards, J. B., Ramsey, S. E., Stuart, G. L., et al. (2002). Evaluation of a behavioral measure of risk taking: The balloon analogue risk task (BART). Journal of Experimental Psychology: Applied, 8, 75-84.

Luria, J. W., Smith, G. A., \& Chapman, J. I. (2000). An evaluation of a safety education program for kindergarten and elementary school children. Archives of Pediatrics \& Adolescent Medicine, 154, 227-231.

MacCallum, R. C., Zhang, S., Preacher, K. J., \& Rucker, D. D. (2002). On 
the practice of dichotomization of quantitative variables. Psychological Methods, 7, 19-40.

Malek, M., Guyer, B., \& Lescohier, I. (1990). The epidemiology and prevention of child pedestrian injury. Accident Analysis \& Prevention, $22,301-313$

Manheimer, D. I., \& Mellinger, G. D. (1967). Personality characteristics of the child accident repeater. Child Development, 38, 491-513.

Matheny, A. P., Jr. (1987). Psychological characteristics of childhood accidents. Journal of Social Issues, 43, 45-60.

Miller, D. C., \& Byrnes, J. P. (1997). The role of contextual and personal factors in children's risk taking. Developmental Psychology, 33, 814823.

National Center for Statistics and Analysis of the National Highway Traffic Safety Administration. (2003). Traffic safety facts 2002: Pedestrians. Retrieved October 5, 2003, from http://www-nrd.nhtsa.dot.gov/departments/ nrd-30/ncsa/AvailInf.html

Piaget, J. (1970). The child's conception of movement and speed. New York: Ballatine.

Pitcairn, T. K., \& Edlmann, T. (2000). Individual differences in road crossing ability in young children and adults. British Journal of Psychology, 91, 391-410.

Pitt, R., Guyer, B., Hsieh, C. C., \& Malek, M. (1990). The severity of pedestrian injuries in children: An analysis of the pedestrian injury causation study. Accident Analysis \& Prevention, 22, 549-559.

Pless, I. B., \& Peckham, C. S. (1986). Risk factors for traffic accidents in childhood. Proceedings of the American Association for Automotive Medicine, 30, 271-283.

Rosenthal, R., \& Rosnow, R. L. (1991). Essentials of behavioral research: Methods and data analysis. Boston: McGraw-Hill.

Rosenthal, R., Rosnow, R. L., \& Rubin, D. B. (2000). Contrasts and effect sizes in behavioral research: A correlational approach. New York: Cambridge University Press.

Rosenthal, R., \& Rubin, D. B. (1982). A simple general purpose display of magnitude in experimental effect. Journal of Educational Psychology, 74, 166-169.

Routledge, D. A. (1975). Observations of children in traffic. Unpublished doctoral dissertation, University of Nottingham, Nottingham, England.

Routledge, D. A., Repetto-Wright, R., \& Howarth, C. I. (1974). The exposure of young children to accident risk as pedestrians. Ergonomics, $17,457-480$.

Sandels, S. (1975). Children in traffic. London: Elek.

Siegler, R. S., \& Richards, D. D. (1979). Development of time, speed, and distance concepts. Developmental Psychology, 15, 288-298.

Sivak, M., Soler, J., \& Tränkle, U. (1989). Cross-cultural difference in driver risk-taking. Accident Analysis \& Prevention, 21, 363-369.

Slovic, P. (1966). Risk-taking in children: Age and sex differences. Child Development, 37, 169-176.

Thomson, J. A. (1991). The facts about child pedestrian accidents. London: Cassell.
Tränkle, U., \& Gelau, C. (1992). Maximization of subjective expected utility or risk control? Experimental tests of risk homeostasis theory. Ergonomics, 35, 7-23.

Trimpop, R. (1994). The psychology of risk taking behavior. Amsterdam: North-Holland.

van der Molen, H. H. (1981). Child pedestrian's exposure, accidents and behavior. Accident Analysis \& Prevention, 13, 193-224.

van Schagen, I. N. L. G. (1988). Training children to make safe crossing decisions. In T. Rothengatter \& R. de Bruin (Eds.), Road user behaviour: Theory and research (pp. 482-489). Assen/Maastricht, the Netherlands: Van Gorcum.

van Schagen, I. N. L. G., \& Rothengatter, T. (1997). Classroom instruction versus roadside training in traffic safety education. Journal of Applied Developmental Psychology, 18, 283-292.

Viney, L. (1971). Accident proneness: Some psychological research. The Medical Journal of Australia, 2, 916-918.

Vinjé, M. P. (1981). Children as pedestrians: Abilities and limitations. Accident Analysis \& Prevention, 13, 225-240.

Vinjé, M. (1982a). A developmental study about anticipation of time intervals in traffic (University of Groningen Traffic Research Centre Report No. VK 82-05). Groningen, the Netherlands: University of Groningen

Vinjé, M. (1982b). Crossing the road: An analysis of the decision-making process in children and adults (University of Groningen Traffic Research Centre Report No. VK 84-04). Groningen, the Netherlands: University of Groningen.

West, R., Train, H., Junger, M., West, A., \& Pickering, A. (1999). Accidents and problem behaviour. The Psychologist, 12, 395-397.

Whitebread, D., \& Neilson, K. (2000). The contribution of visual search strategies to the development of pedestrian skills by 4-11 year-old children. British Journal of Educational Psychology, 70, 539-557.

Wilson, D. G., \& Grayson, G. B. (1980). Age-related differences in the road crossing behavior of adult pedestrians (Research report). Crowthorne, Berkshire, England: Transport and Road Research Laboratory.

Young, D. S., \& Lee, D. N. (1987). Training children in road crossing skills using a roadside simulation. Accident Analysis \& Prevention, 19, 327 341.

Zeedyk, M. S., Wallace, L., Carcary, B., Jones, K., \& Larter, K. (2001). Children and road safety: Increasing knowledge does not improve behaviour. British Journal of Educational Psychology, 71, 573-594.

Zeedyk, M. S., Wallace, L., \& Spry, L. (2002). Stop, look, listen, and think? What young children really do when crossing the road. Accident Analysis \& Prevention, 34, 43-50.

Received June 2, 2002

Revision received August 22, 2003

Accepted August 22, 2003

\section{Instructions to Authors}

For Instructions to Authors, please consult the June 2003 issue or visit www.apa.org/journals/xap and click on Submission Guidelines. 
Copyright of Journal of Experimental Psychology / Applied is the property of American Psychological Association and its content may not be copied or emailed to multiple sites or posted to a listserv without the copyright holder's express written permission. However, users may print, download, or email articles for individual use. 